\title{
ERLIQUIOSE CANINA PRECEDIDA POR DESCOLAMENTO DE RETINA: RELATO DE CASO
}

\author{
Valéssia Kelly Miranda MAIA $^{1 *}$; José Andreey Almeida TELES ${ }^{2}$; Gil Dutra FURTADO ${ }^{3}$
}

${ }^{1}$ Pós-Graduanda do Curso de Clínica Médica e Cirúrgica de Pequenos Animais/Centro Universitário CESMAC, Maceió, Alagoas, Brasil.

${ }^{2}$ Mestre em Biociência Animal, Médico Veterinário, Professor/Centro Universitário Maurício de Nassau (UNINASSAU), João Pessoa, Paraíba, Brasil.

${ }^{3}$ Graduando em Medicina Veterinária/Centro Universitário Maurício de Nassau (UNINASSAU), João Pessoa, Paraíba; Agrônomo/Universidade Federal da Paraíba (UFPB); Doutor em Psicobiologia/Universidade Federal do Rio Grande do Norte (UFRN); Agrônomo-Sócio da Cooperativa do Agronegócio (COOPAGRO), Natal, Rio Grande do Norte, Brasil.

*Autor para correspondência. E-mail: valessiakelly@ hotmail.com

Resumo. No dia 21-03-16, foi atendida no consultório veterinário da "VM RURAL" uma cadela, raça labrador, idade 3 anos, pesando $20 \mathrm{~kg}$, com erliquiose canina que culminou num descolamento de retina causando cegueira. O método diagnóstico utilizado foi o clínico, associado ao diagnóstico laboratorial através do hemograma e pesquisa de hemoparasitos que auxiliou na confirmação desta doença. A conduta terapêutica baseou-se na administração de doxiciclina, na dosagem de $10 \mathrm{mg} / \mathrm{kg}$, via oral, durante 30 dias, uma vez ao dia. Posteriormente associou com dipropionato de imidocarb na dose de $5 \mathrm{mg} / \mathrm{kg}$ subcutânea, seguida de outra administração duas semanas depois. Com a administração da doxiciclina o paciente reagiu bem, mas o hematócrito não atingia o valor referencial, após as duas aplicações do imidocarb o paciente continuou estável e o hematócrito atingiu a concentração desejada. A efetivação desse estudo pretende alertar para a gravidade dos sinais oftálmicos causados pela erliquiose, objetivando assim o diagnóstico precoce, para que o paciente seja tratado corretamente e em tempo hábil, evitando quadros mais graves como o que ocorreu com esse paciente acima mencionado. Além da conscientização da importância da prevenção, principalmente em locais de grande concentração de animais que possui alta chance de infestação por carrapatos, um complicante é a inexistência de vacina contra esta enfermidade. A erliquiose canina deve ser sempre pesquisada e alvo de estudo, por ser uma doença cosmopolita, por dispor de um vetor de difícil erradicação e por possuir importância na saúde pública, já que nas últimas décadas passou a ser considerada uma zoonose.

Palavras-Chave: Ehrlichia canis; Oftalmopatias; Carrapato.

CANINE EHRLICHIOSIS PRECEDED BY RETINAL DETACHMENT: A CASE REPORT 
Abstract. In the day 21-03-16, was met at the veterinarian's Office "VM RURAL" a dog, breed labrador, age 3 years, weighing $20 \mathrm{~kg}$, with canine ehrlichiosis that culminated in a detached retina causing blindness. The diagnostic method used was the clinical, associated to laboratorial diagnosis through the blood count and hemoparasite search which assisted in the confirmation of this disease. The therapeutic conduct was based on the administration of doxycycline, in $10 \mathrm{mg} / \mathrm{kg}$, oral administration, during 30 days, once a day. Posteriorly was associated with imidocarb dipropionate on $5 \mathrm{mg} / \mathrm{kg}$ subcutaneous dose, followed by another two weeks after administration. With the administration of doxycycline the patient reacted well, but the hematocrit did not reached the reference value, after those two applications of imidocarb the patient continued stable and the hematocrit has reached the desired concentration. The execution of this study intends to draw attention to the gravity of the ophthalmic signs caused by ehrlichiosis, aiming the early diagnosis, for which the patient is treated correctly and in a timely manner, avoiding serious frameworks like what happened with this patient mentioned above. Besides the awareness of the importance of prevention, mainly in places of great concentration of animals with high chance of infestation by ticks, a complicating factor is the absence of vaccine against this disease. The canine ehrlichiosis should always be investigated and subjected to study, for being a cosmopolitan disease, and having a hard vector eradication and for its importance in public health, since in recent decades it has become considered a zoonosis.

Keywords: Ehrlichia canis; Oftalmopathy; Tick.

\section{ERLRLIOSIS CANINA PRECEDIDA POR LA DESMONTAJE RETINAL: UN INFORME DEL CASO}

Resumen. En el día 21-03-16, se encontró en la oficina del veterinario "VM RURAL" un perro, raza labrador, de 3 años de edad, con un peso de $20 \mathrm{~kg}$, con erliquiosis canina que culminó en una retina desprendida que causó ceguera. El método de diagnóstico utilizado fue el clínico, asociado al diagnóstico de laboratorio a través del hemoparásito y la búsqueda de hemoparásitos, lo que ayudó a confirmar esta enfermedad. La conducta terapéutica se basó en la administración de doxiciclina, en 10 $\mathrm{mg} / \mathrm{kg}$, administración oral, durante 30 días, una vez al día. Posteriormente se asoció con dipropionato de imidocarb en dosis subcutánea de $5 \mathrm{mg} / \mathrm{kg}$, seguido de otras dos semanas después de la administración. Con la administración de doxiciclina, el paciente reaccionó bien, pero el hematocrito no alcanzó el valor de referencia, después de esas dos aplicaciones de imidocarb, el paciente continuó estable y el hematocrito alcanzó la concentración deseada. La ejecución de este estudio pretende llamar la atención sobre la gravedad de los signos oftálmicos causados por la ehrlichiosis, con el objetivo del diagnóstico temprano, para el cual el paciente es tratado correctamente y de manera oportuna, evitando marcos graves como lo que sucedió con este paciente mencionado anteriormente. Además de la conciencia de la importancia de la prevención, principalmente en lugares de gran concentración de animales con alta probabilidad de infestación por garrapatas, un factor complicado es la ausencia de 
vacuna contra esta enfermedad. La ehrlichiosis canina siempre debe investigarse y someterse a estudio, por ser una enfermedad cosmopolita y por tener una erradicación dura de vectores y por su importancia en la salud pública, ya que en las últimas décadas se ha considerado una zoonosis.

Palabras Clave: Ehrlichia canis; Oftalmopatía; Garrapata.

\section{INTRODUÇÃO}

A erliquiose canina é uma moléstia riquetsial infecciosa que geralmente se caracteriza por redução dos elementos sanguíneos. A principal espécie que acomete os cães é a Ehrlichia canis, e possui o carrapato Rhipicephalus sanguineus como vetor e reservatório da enfermidade, que pela picada de um carrapato infectado configura-se como a principal forma de transmissão da enfermidade (SAITO, 2009).

Os sinais clínicos frequentemente são inespecíficos, e os proprietários mais desatentos podem não perceber o início da doença, este é um fator limitante, pois além de sinais não patognomônicos, destaca-se ainda alterações oftálmicas que podem se agravar com o tardamento do diagnóstico,apresentando assim neurite óptica, hemorragias e descolamento da retina (por massa hemorrágica sub retinal ou por exsudatos) que por sua vez gera uma perda de visão irreversível (KOMNENOU et al., 2007).

Apesar da severidade que a enfermidade pode alcançar o tratamento é relativamente simples, obtendo resultados satisfatórios com o uso da doxiciclina,além da reversão da opacidade corneana que acontece nos primeiros sinais oculares (ORIÁ et al., 2008).

A prevenção da doença tem um caráter de suma importância, nos canis e nos locais de grande concentração de animais, sendo realizada através do controle do vetor da doença: o carrapato. Para tanto, produtos acaricidas ambientais e de uso tópico são eficazes desde que seja realizado o manejo correto (WOODY et al., 1991).

A erliquiose canina deve estar em foco de pesquisa e estudo, por ser uma doença de prevalência em todo território nacional, por obter um vetor de difícil erradicação e por possuir importância na saúde pública, já que nas últimas décadas passou a ser considerada uma zoonose. O diagnóstico precoce é a maior ferramenta para o tratamento da erliquiose canina, pois quando diagnosticada no início dos sintomas, tem grande chance de cura e bom prognóstico (SILVA, 2015).

O estudo de caso objetivou alertar e prover maiores esclarecimentos aos médicos veterinários e criadores de cães, sobre os sinais oculares que acontecem na erliquiose canina que por muita vezes passam despercebidas, mesmo sendo consideradas alterações comuns nesta 
doença. Em consequência da falta de diagnóstico precoce, o paciente pode sofrer danos irreversíveis na visão, como ocorrido neste estudo de caso.

\section{REVISÃO DE LITERATURA}

A erliquiose é uma das principais doenças infectocontagiosas, causada por um hemoparasita da ordem Rickettsiales e do gênero Ehrlichia spp.,são parasitas intracelulares obrigatórios de células hematopoiéticas maduras ou imaturas. A principal espécie que acomete os cães é a Ehrlichia canis, epossui o carrapato Rhipicephalus sanguineuscomo vetor e reservatório da enfermidade, outra maneira de transmissão da enfermidade, esta bem menos comum, é por meio da transfusão sanguínea, pelo sangue infectado de um cão para outro sadio (SILVA, 2015).

A erliquiose canina é uma doença mundialmente distribuída em várias regiões geográficas, as quais incluem sudeste da Ásia, a África, a Europa, a Índia, a América Central e a América do Norte. No Brasil, foi relatada pela primeira vez em Belo Horizonte, Minas Gerais (COSTAet al., 1973). A maior prevalência no país é observada na região Nordeste (43\%) e a menor na região Sul (1,70\%). A frequência desta espécie de carrapato, encontrado naturalmente infectados pela E. canisno Brasil, tem variado de 2,3\% a 6,2\% (AGUIAR et al., 2007).

A erliquiose vem sendo identificada como causa de morbidade e mortalidade nos animais e no homem, representando uma importante zoonose, em decorrência da maior exposição humana a locais onde a presença de carrapatos se faz presente e em regiões onde a erliquiose canina é enzoótica (MORAES et al., 2004; SAITO, 2009).Animais de todas as raças e idades são acometidos, inclusive fêmeas gestantes, onde o feto poderá ter má formação e a fêmea prenhe poderá vir a óbito devido àhemorragia oriunda de trombocitopenia(ROBERTS et al., 1986).

Os sinais clínicos frequentemente são inespecíficos, e os proprietários mais desatentos podem não perceber o início da doença. Durante a fase aguda, que dura em média, duas a quatro semanas, destacam-se febre, perda de peso, anorexia, apatia, linfadenopatia, vasculite, depressão e alterações neurológicas, oftálmicas e musculares e também poliartrite. (KUEHN \& GAUNT, 1985; ENG \& GILLES, 1989). Dentre os sinais oftalmológicos, que são considerados manifestações comuns da doença, as síndromes mais relatadas são as conjuntivites, as alterações da córnea, o glaucoma, a catarata, a uveíte bilateral anterior, a coriorretinite, o papiledema, as hemorragias retinianas e as esclerite necrosante. Entre os sinais mais comuns encontram-se o blefaroespasmo e a fotofobia (TEIXEIRA PINTO; CARVALHO, 2013). 
As retinopatias apresentam-se de várias formas nas alterações graves: neurite óptica, hemorragias e descolamento da retina (por massa hemorrágica sub retinal ou por exsudados). As lesões na retina evidenciam o ingurgitamento e a tortuosidade vascular, a presença de infiltrados perivasculares, a retinite difusa, a hemorragia retiniana, o descolamento retiniano e as cicatrizes coriorretinianas com atrofia da retina (KOMNENOU et al., 2007).Frequentes hemorragias podem induzir o descolamento da retina de forma secundária(EVERMANN, 1998; MARTIN, 1999).

Os achados laboratoriais frequentemente observados são leucopenia discreta, anemia e trombocitopenia,que pode ser causada pela destruição imunológica periférica das plaquetas, pelo aumento do sequestro das mesmas pelo baço ou aumento no consumo de plaquetas pelo endotélio inflamado. Após a fase aguda, o paciente pode se curar ou a doença pode evoluir para fase subclínica, nessa fase o paciente geralmente não apresenta sinais clínicos, contudo, o parasito ainda persiste no organismo, essa fase pode durar anos (DAGNONE et al., 2001; SOUSA et al., 2010; ISOLA et al., 2012).

Os cães com boa resposta imunológica eliminarão o parasita, enquanto os cães imunossuprimidos irão desenvolver a fase crônica da doença. Na fase crônica ocorre perda de peso, palidez de mucosas, pancitopenia com comprometimento da medula óssea, óbito por hemorragias causadas pela trombocitopenia e trombocitopatias ou ainda infecções secundárias (DAGNONE et al., 2001; SOUSA et al., 2010; ISOLA et al., 2012).

O diagnóstico é feito tanto através dos sinais clínicos, como pelas alterações laboratoriais observadas no hemograma, sendo a anemia e a trombocitopenia as mais evidentes. Muitas vezes, durante o exame de sangue, o patologista clínico veterinário visualiza o agente (as chamadas mórulas) no interior dos neutrófilos, o achado destas estruturas fecha o diagnóstico de maneira clara e definitiva, mas a não observância delas não descarta a enfermidade. A PCR (reação em cadeia da polimerase) é uma técnica de diagnóstico extremamente eficaz, já que detecta o material genético da riquetsia no sangue do hospedeiro.Os sinais e sintomas oculares na erliquiose canina são relevantes no diagnóstico diferencial para outras causas de oftalmopatias, pois desta forma, após o diagnóstico, proporcionará ao paciente canino o correto tratamento contra a doença através de medicamentos específicos permitindo assim a resolução parcial ou completa das lesões oculares (ALVES et al.; 2004).

Apesar da severidade que a enfermidade pode alcançar o tratamento é relativamente simples, consiste na administração de antibióticos; sendo a doxiciclina o antibiótico de escolha em todas as suas fases, além de tratamento suporte que inclui transfusões sanguíneas é 
importante à precaução de verificar se o sangue recebido é do mesmo tipo para que não haja rejeição do organismo e acabe agravando ainda mais o quadro do animal (em casos de anemia e trombocitopenia importantes), fluidoterapia, protetores gástricos e hepáticos (DAMAS, 2012).

A doxiciclina além de ser absorvida com rapidez quando administrada por via oral, possibilita uma menor taxa de recidiva comparativamente às outras tetraciclinas. A eficácia da droga no tratamento da erliquiose na dose de $10 \mathrm{mg} / \mathrm{Kg} /$ dia em dose única foi demostrada por vários autores (BREITSCHWERDT, 2004; WOODY; HOSKINS, 1991). Em pacientes com insuficiência renal, devido à via pela qual é eliminada (fecal), as concentrações da doxiciclina não tendem a aumentar no sangue, sendo assim ideal para tratar infecções suscetíveis quando a insuficiência renal for um fator complicante, como em infecções por E. canis (ALMOSNY, 2002; ADAMS, 2003).

O dipropionato de imidocarb (IMIZOL), administrado em dosagem de $5 \mathrm{mg} / \mathrm{kg}$, via subcutânea, com intervalode quinze dias, é altamente efetivo em cães com erliquiose refratária e em cães com infecções mistas por Ehrlichia canis e a Babesia canis (COUTO, 1998).

O prognóstico depende da fase em que a doença for diagnosticada e do início da terapia, quanto antes se inicia o tratamento nas fases agudas, melhor o prognóstico. Entretanto, na fase subclínica, o prognóstico é de favorável a reservado, já que afeta cães assintomáticos ou com risco de desenvolverem a fase crônica. Muitos animais tratados permanecem como portadores da doença, podendo dessa forma ocorrer recidivas (JONES, 2000).

A prevenção da doença tem um caráter de suma importância nos canis e nos locais de grande concentração de animais, devido à inexistência de vacina contra esta enfermidade, a prevenção é realizada através do controle do vetor da doença: o carrapato. Para tanto, produtos acaricidas ambientais e de uso tópico são eficazes desde que seja realizado o manejo correto (WOODY et al., 1991).

\section{RELATO DE CASO}

Foi atendida em 21-03-16 no consultório Veterinário da "VM RURAL", em Canindé de São Francisco-SE, uma cadela de nome Ariel, da raça Labrador, 03 anos de idade e 20kg de peso, apresentando opacidade corneanabilateral, lacrimejamentoe sensibilidade à luz. Ao exame físico não apresentou nenhuma alteração importante, demonstrou-se em alerta e saudável. $\mathrm{O}$ paciente foi conduzido a um oftalmologista veterinário que solicitou um hemograma, testes diferenciais (Dirofilariose+Lyme + Ehrlichia + Anaplasma) e o teste de fundo de olho. O hemograma resultou numa anemia normocítica normocrômica, trombocitopenia e leucopenia 
por linfopenia. No teste diferencial foi positivo para erliquiose. $O$ teste de fundo de olhoconfirmou o descolamento de retina bilateral, resultando numa cegueira irreversível.

Diante desses resultados, a conduta terapêutica embasou-se na administração de Cloridrato de Doxiciclina, $5 \mathrm{mg} / \mathrm{kg}$ a cada 12 horas, por via oral, durante 21 dias seguidos. No $18^{\circ}$ dia, o animal apresentava-se clinicamente normal, normoréxico, foi realizada uma nova avaliação hematológica, que demonstrou uma piora no quadro hematológico. Então foi prescrito Cloridrato de Doxiciclina, $10 \mathrm{mg} / \mathrm{Kg}$, uma vez ao dia, por via oral, durante 30 dias, associado ao Imidocarb, $5 \mathrm{mg} / \mathrm{Kg}$, em duas dosescom intervalo de 15 dias precedido por 0,02 $\mathrm{mg} / \mathrm{Kg}$ de Atropina, ambos por via subcutânea.

Após 18 dias de tratamento, foi realizada nova avaliação hematológica do animal, onde se observou a normalização dos resultados, continuando com o aspecto físico normal, concluindo que o tratamento foi bem sucedido. Oanimal apresentou cura evidente e demonstrou uma boa adaptação a perda da visão.

\section{DISCUSSÃO}

Aerliquiose é uma hemoparasitose frequente em cães de todas as idadese não tem predisposição racial, necessitando somente da infestação de carrapato ou transfusão com sangue contaminado para instituir a enfermidade (ROBERTS et al., 1986).

A gravidade do envolvimento ocular nesta doença varia de paciente para paciente, podendo gerar casos graves como hemorragia subretiniana, levando ao descolamento da retina e à cegueira, corroborando com os achados deste estudo (KOMNENOU et al.; 2007).

A uveíte anterior foi àdoença ocular mais encontrada na erliquiose canina no estudo de (TEIXEIRA PINTO; CARVALHO, 2013) em contrapartida a panuveíte com descolamento de retina foi à lesão ocular observada com mais frequência no estudo realizado em Barcelona, Espanha, por Leiva (LEIVA, et al., 2005). As razões para essa diferença não são citadas, sabendo-se que o diagnóstico correto da doença é importante principalmente na fase crônica, para que se institua o tratamento específico permitindo assim a resolução parcial ou completa das lesões oculares e evitando óbitos.

A eficácia da doxiciclina no tratamento da erliquiose na dose de $10 \mathrm{mg} / \mathrm{Kg} /$ dia, durante 30 dias foi demostrada por vários autores (BREITSCHWERDT, 2004; WOODY; HOSKINS, 1991). O dipropionato de imidocarb (IMIZOL), administrado em dosagem de $5 \mathrm{mg} / \mathrm{kg}$, via subcutânea, com intervalode quinze dias, é altamente efetivo em cães com erliquiose refratária por Ehrlichiacanis (COUTO, 1998) confirmando os resultados do tratamento deste estudo. 
Segundo Brandão e Hagiwara (2002), apesar do dipropionato de imidocarb ser efetivo tanto para a E. canisquanto para B.canis, os seus efeitos colaterais representam a principal desvantagem do seu uso, tais como salivação intensa, secreção ocular serosa, diarréia, dispnéia ou depressão. Essas reações podem ocorrer dez a doze horas após a sua administração, sendo indicado um pré-tratamento com sulfato de atropina $(0,02$ a $0,04 \mathrm{mg} / \mathrm{kg}$ via endovenosa) para evitar o surgimento de tais efeitos anticolinérgicos, ratificando o tratamento utilizado neste estudo.

\section{CONSIDERAÇÕES FINAIS}

Houve um tardamento no diagnóstico que implicou no agravamento da doença, desencadeando alterações oftálmicas irreversíveis. Mediante esta experiência é possível considerar antecipadamente a erliquiose canina como suspeita diagnóstica, principalmente quando houver histórico de infestação por carrapatos associado a sinais oftálmicos, tendo grandes chances de cura e bom prognóstico. Deve sempre ser sugerida a prevenção dessa afecção, que é feita como cuidado no local onde o animal vive mantendo limpo e arejado e utilizar coleiras repelentes.

\section{REFERÊNCIAS}

ADAMS, H.R. Farmacologia e terapêutica em veterinária. 8. ed. Rio de Janeiro: Guanabara Koogan, 2003.

AGUIAR, D.M.; SAITO, T.B.; HAGIWARA, M.K.; MACHADO, R.Z.; LABRUNA, M.B. Diagnóstico sorológico de erliquiose canina com antígeno brasileiro de Ehrlichia canis. Ciência Rural, 2007.

ALMOSNY, N.R.P. Hemoparasitoses em pequenos animais domésticos e como zoonoses. Rio de Janeiro: NDLF Livros, 2002.

ALVES, M.L.; LINHARES, G.F.C.; CHAVES, N.S.T.; MONTEIRO, L.C.; LINHARES, D.C.L. Avaliação de Indicadores e Protocolo para o Diagnóstico da Pancitopenia Tropical Canina por PCR. Ciência Animal Brasileira, p. 49-54, 2004.

BRANDÃO, L.P.; HAGIWARA, M.K. Babesiose canina - revisão: Clínica Veterinária. SãoPaulo: Ed. Guará, 2002. 
BREITSCHWERDT, E.B. Riquetsioses. In: ETTINGER, S.J.; FELDMAN, E.C. (Org.). Tratado de medicina interna veterinária: doenças do cão e do gato. 5. ed. Rio de Janeiro: Guanabara Koogan, 2004. p. 422-429.

COSTA, J.O.; BATISTA Jr., J.A.; SILVA, M.; GUIMARÃES, M.P. Ehrlichia canis infection in dog in Belo Horizonte - Brazil. Arquivo da Escola de Veterinária da Universidade Federal de Minas Gerais, Belo Horizonte, v. 25, n. 2, p. 199-206, 1973.

COUTO, C.G. Doenças rickettsiais. In: BICHARD, S.J.: SHERDING, R.G. (Org.). Manual Saunders: clínica de pequenos animais. Rio de Janeiro: Roca, 1998. p. 139-142.

DAGNONE, A.S.; MORAIS, H.S.A.; VIDOTTO, O. Animal and human Ehrlichiosis. Semina: Ciências Agrárias, Londrina, v. 22, n. 2, p. 191-201, 2001.

DAMAS, J.A.K. Erliquiose Canina: Revisão de Literatura. Curso de pós-graduação em clínica médica e cirúrgica de pequenos animais, UNIP- Universidade Paulista, Vitória, 2012.

ENG, T.R.; GILLES, R. Ehrlichiosis (Zoonosis Update). Journal of the American Veterinary Medical Association, Schaumburg, v. 194, n. 4, p. 497-500, 1989.

EVERMANN, J.F. Laboratory diagnosis of viral and rickettsial infections. In: GREENE, C.E. (Ed.). Infectious diseases of the dog and cat. 2. ed. Philadelphia: WB Saunders, 1998. p 1-61.

ISOLA, J.G.M.P.; CADIOLI, F.A.; NAKAGE, A.P. Erliquiose canina - Revisão de Literatura. Revista Científica Eletrônica de Medicina Veterinária, São Paulo, v. 9, n. 18, p. 1-11, 2012.

JONES, T.C.; HUNT, R.D.; KING, N.W. Patologia Veterinária. 6. ed. São Paulo: Editora Manole, 2000.

KOMNENOU, A.; MYLONAKIS, M.; KOUTI, V.; TENDOMA, L.; LEONTIDES, L.; SKOUNTZOU, E.; DESSIRIS, A.; KOUTINAS, A.; OFRRI, R. Ocular manifestations of natural canine monocytic ehrlichiosis (Ehrlichia canis): a retrospective study of 90 cases. Veterinary Ophthalmology, Medford, v. 10, p. 137-142, 2007. 
KUEHN, N.F.; GAUNT, S.D. Clinical and hematologic findings in canine ehrlichiosis. Journal of the American Veterinary Medical Association, v. 186, n. 4, p. 355-358, 1985.

LEIVA, M.; NARANJO, C.; PEÑA, M.T. Ocular signs of canine monocytic ehrlichiosis: a retrospective study in dogs from Barcelona, Spain. Veterinary Ophthalmology, Medford, v. 8, n. 6, p. 387-393, 2005.

MARTIN, C.L. Ocular manifestations of systemic disease. In: GELATT, K.N. (Ed.). Veterinary ophthalmology. Philadelphia: Lippincott Williams \& Wilkins, 1999. p. 14081409.

MORAES, H.A.; ALMOSNY, N.R.P.; LABARTHE, N. Diretrizes gerais para diagnóstico e manejo de cães infectados por Ehrlichia spp. Clínica Veterinária, São Paulo, v. 9, n. 48, p. 2830, 2004.

ORIÁ, A.P.; DÓREA NETO, F.A.; MACHADO, R.Z.; SANTANA, A.E.; GUERRA, J.L.; SILVA, V.L.D.; BEDFORD, P.G.C.; LAUS, J.L. Ophthalmic, hematologic and serologic findings in dogs with suspected Ehrlichia canis infections. Revista Brasileira de Ciência Veterinária, v. 15, n. 2, p. 94-97, 2008.

ROBERTS, S.J. Veterinary obstetrics and genital diseases theriogenology. 3. ed. 1986.

SAITO, T.B. Estudo da erliquiose em cães expostos a carrapatos Rhipicephalus sanguineus experimentalmente infectados. Tese (Doutorado) - Faculdade de Medicina Veterinária e Zootecnia, Universidade de São Paulo. São Paulo, 2009.

SILVA, I.P.M. Ehrlichiosis canine - literature review. Revista Científica de Medicina Veterinária, São Paulo, v. 13, n. 24, 2015.

SOUSA, V.R.F.; ALMEIDA, A.B.P.F.; BARROS, L.A.; SALES, K.G.; JUSTINO, C.H.S.; DALCIN, L.; BOMFIM, T.C.B. Clinical and molecular evaluation of dogs with ehrlichiosis. Ciência Rural, Santa Maria, v. 40, n. 6, p. 1309-1313, 2010. 
TEIXEIRA PINTO, A.B.; CARVALHO, C.B. Eye diseases in canine monocytic ehrlichiosis. Jornal Brasileiro de Ciência Animal, v. 6, n. 12, 442-452, 2013.

WOLDEHIWET, Z.; RISTIC, M. Rickettsial and chlamydial diseases of domestic animals. Oxford: Pergamon Press. 1993.

WOODY, B.J.; HOSKINS, J.D. Ehrlichial diseases of dogs. Veterinary Clinics of North America: Small Animal Practice, Maryland Heights, v. 21, n. 1, p. 75-98, 1991. 